\title{
The 4D SU(3) gauge theory with an imaginary $\theta$ term
}

\author{
Haralambos Panagopoulos \\ Department of Physics, University of Cyprus, Lefkosia, CY-1678, Cyprus \\ E-mail: haris@ucy.ac.cy
Ettore Vicari
Dipartimento di Fisica, Università di Pisa and INFN, I-56127 Pisa, Italy
E-mail: vicari@df.unipi.it

\begin{abstract}
:
We study the scaling behavior of the $4 \mathrm{D} \mathrm{SU}(3)$ lattice gauge theory in the presence of a $\theta$ term, by Monte Carlo simulations computing the topological properties at imaginary $\theta$. The numerical results provide a good evidence of scaling in the continuum limit. The imaginary $\theta$ dependence of the ground-state energy turns out to be well described by the first few terms of related expansions around $\theta=0$, providing accurate estimates of the first few coefficients, up to $O\left(\theta^{6}\right)$.
\end{abstract}

KEYwords: Gauge Field Theories, Lattice Gauge Field Theories, Theta Term, Topological Term. 
Four-dimensional $S U(N)$ gauge theories have a nontrivial dependence on the parameter $\theta$ which appears in the Euclidean Lagrangian as

$$
\mathcal{L}_{\theta}=\frac{1}{4} F_{\mu \nu}^{a}(x) F_{\mu \nu}^{a}(x)-i \theta \frac{g^{2}}{64 \pi^{2}} \epsilon_{\mu \nu \rho \sigma} F_{\mu \nu}^{a}(x) F_{\rho \sigma}^{a}(x),
$$

where

$$
q(x)=\frac{g^{2}}{64 \pi^{2}} \epsilon_{\mu \nu \rho \sigma} F_{\mu \nu}^{a}(x) F_{\rho \sigma}^{a}(x)
$$

is the topological charge density. The $\theta$ dependence of the ground-state energy density $F(\theta)$, defined as

$$
Z_{\theta}=\int[d A] \exp \left(-\int d^{4} x \mathcal{L}_{\theta}\right)=\exp [-V F(\theta)]
$$

where $V$ is the space-time volume, can be written as

$$
\mathcal{F}(\theta) \equiv F(\theta)-F(0)=\frac{1}{2} \chi \theta^{2} s(\theta)
$$

where $\chi$ is the topological susceptibility at $\theta=0$,

$$
\chi=\int d^{4} x\langle q(x) q(0)\rangle_{\theta=0}=\frac{\left\langle Q^{2}\right\rangle_{\theta=0}}{V}, \quad Q \equiv \int d^{4} x q(x),
$$

and $s(\theta)$ is a dimensionless even function of $\theta$ such that $s(0)=1$. Assuming analyticity at $\theta=0, s(\theta)$ can be expanded as

$$
s(\theta)=1+b_{2} \theta^{2}+b_{4} \theta^{4}+\cdots
$$

where only even powers of $\theta$ appear. Large- $N$ scaling arguments [1, 2, 3] applied to the Lagrangian (1) of a $4 \mathrm{D} \mathrm{SU}(N)$ gauge theory indicate that the relevant scaling variable in the large- $N$ limit is $\bar{\theta} \equiv \theta / N$. This implies that in the large- $N$ limit $\chi=O(1)$, while the coefficients $b_{2 i}$ are suppressed by powers of $N$, i.e. [4]

$$
b_{2 i}=O\left(N^{-2 i}\right)
$$

The presence of the $\theta$ term has important phenomenological consequences, since it violates parity and time reversal symmetry. Experimental bounds on the $\theta$ parameter in QCD are best obtained from the electric dipole moment of the neutron [5, 6, 团, which leads to an unnaturally small value for $\theta,|\theta| \lesssim 10^{-10}$. This suggests the idea that there must be a mechanism responsible for suppressing the value of $\theta$ in the context of QCD. However, the issue of the $\theta$ dependence, even within pure gauge theory, has phenomenological relevance because it provides an explanation to the so-called $\mathrm{U}(1)_{A}$ problem [7, 8, 9], i.e. explaining the large mass of the $\eta^{\prime}$ meson with respect to the $\pi$ meson. The $\theta$ dependence of $4 \mathrm{D} \mathrm{SU}(N)$ gauge theories is particularly interesting in the large- $N$ limit where the issue may also be addressed by other 
approaches, such as AdS/CFT correspondence applied to nonsupersymmetric and non conformal theories, see e.g. Refs. 110, 11, 12, 13, 14.

Due to the nonperturbative nature of the $\theta$ dependence, quantitative assessments of this issue have largely focused on the lattice formulation of the theory, using Monte Carlo (MC) simulations. However, the complex nature of the $\theta$ term in the Euclidean QCD Lagrangian prohibits a direct $\mathrm{MC}$ simulation at $\theta \neq 0$. Information on the $\theta$ dependence of physically relevant quantities, such as the ground state energy and the spectrum, has been obtained by computing the coefficients of the corresponding expansions around $\theta=0$. The coefficients of expansion of $s(\theta)$, cf. Eq. (6), can be determined from appropriate zero-momentum correlation functions «4, 15] of the topological charge density at $\theta=0$, which are related to the moments of the $\theta=0$ probability distribution $P(Q)$ of the topological charge $Q$. Indeed

$$
\begin{aligned}
b_{2} & =-\frac{\chi_{4}}{12 \chi}, \quad \chi_{4}=\frac{1}{V}\left[\left\langle Q^{4}\right\rangle-3\left\langle Q^{2}\right\rangle^{2}\right]_{\theta=0}, \\
b_{4} & =-\frac{\chi_{6}}{360 \chi}, \quad \chi_{6}=\frac{1}{V}\left[\left\langle Q^{6}\right\rangle-15\left\langle Q^{2}\right\rangle\left\langle Q^{4}\right\rangle+30\left\langle Q^{2}\right\rangle^{3}\right]_{\theta=0},
\end{aligned}
$$

etc. They parameterize the deviations of $P(Q)$ from a simple Gaussian behavior. As shown in Ref. [16], the correlation functions involving multiple zero-momentum insertions of the topological charge density can be defined in a nonambiguous, regularization independent way, and therefore the expansion coefficients $b_{2 i}$ are well defined renormalization group invariant quantities. The numerical evidence for a nontrivial $\theta$-dependence, obtained through $\mathrm{MC}$ simulations of the lattice formulation, appears quite robust. We refer the reader to Ref. [4] for a recent review. On the other hand, $\mathrm{MC}$ simulations at $\theta=0$ have only made it possible to estimate the ground-state energy up to the $O\left(\theta^{4}\right)$ term, because the statistical errors rapidly increase with increasing order of the expansion, essentially for importance sampling problems. The large- $N$ prediction $b_{2}=O\left(N^{-2}\right)$ has been already supported by numerical results [15, 17, 18]. The calculation of the higher-order terms would provide a further check of the large- $N$ arguments, which predict them to be suppressed by higher powers of $N$, as in Eq. (7).

In this paper we consider imaginary values of $\theta$, which make the Euclidean Lagrangian (西) real, thus allowing us to investigate the imaginary $\theta$ dependence by $\mathrm{MC}$ simulations. Assuming analyticity at $\theta=0$, the results may provide quantitative information on the expansion around $\theta=0$, cf. Eqs. (4) and (6). Indeed, fits of the data to polynomials of imaginary $\theta$ may provide more accurate estimates of the coefficients, overcoming the rapid increasing of the statistical errors observed at $\theta=0$. Another interesting issue concerns the continuum limit of the theory for generic, and in particular imaginary, $\theta$ values. Perturbative renormalization-group (RG) arguments [19] indicate that $\theta$ is a $R G$ invariant parameter of the theory, thus the continuum limit should be approached while keeping $\theta$ fixed to any complex 
value. Although reflection positivity [20], and therefore a well defined relativistic field theory, requires real $\theta$ values, imaginary $\theta$ may still give rise to a well defined continuum limit in the sense of a statistical field theory, with the same asymptotic behavior controlled by the perturbative $\beta$ function which does not depend on $\theta$. As we shall see, this is indeed supported by the numerical data for the $4 \mathrm{D} \mathrm{SU}(3)$ lattice gauge theory, at least for $|\theta|<\pi$, which are well described by the first few nontrivial terms of the expansion around $\theta=0$.

Introducing the real parameter $\theta_{i}$, defined by

$$
\theta \equiv-i \theta_{i}
$$

Eq. (四) becomes

$$
\Phi\left(\theta_{i}\right) \equiv \mathcal{F}\left(-i \theta_{i}\right)=-\frac{1}{2} \chi \theta_{i}^{2} s\left(-i \theta_{i}\right)=-\frac{1}{2} \chi \theta_{i}^{2}\left(1-b_{2} \theta_{i}^{2}+b_{4} \theta_{i}^{4}+\cdots\right) .
$$

We thus obtain

$$
\begin{aligned}
& \frac{\langle Q\rangle_{\theta_{i}}}{V}=-\frac{\partial \Phi\left(\theta_{i}\right)}{\partial \theta_{i}}=\chi \theta_{i}\left(1-2 b_{2} \theta_{i}^{2}+3 b_{4} \theta_{i}^{4}+\cdots\right), \\
& \frac{\left\langle Q^{2}\right\rangle_{\theta_{i}}^{c}}{V} \equiv \frac{\left\langle Q^{2}\right\rangle_{\theta_{i}}-\langle Q\rangle_{\theta_{i}}^{2}}{V}=-\frac{\partial^{2} \Phi\left(\theta_{i}\right)}{\partial \theta_{i}^{2}}=\chi\left(1-6 b_{2} \theta_{i}^{2}+15 b_{4} \theta_{i}^{4}+\cdots\right),
\end{aligned}
$$

etc.

The $4 \mathrm{D} \mathrm{SU}(N)$ gauge theory with an imaginary $\theta$ term can be nonpertubatively formulated on the lattice by

$$
\begin{aligned}
& Z_{L}=\int[d A] \exp \left(-S_{L}+\theta_{L} Q_{L}\right), \\
& S_{L}=-\frac{\beta}{N} \sum_{x, \mu>\nu} \operatorname{Re} \operatorname{Tr} \Pi_{\mu \nu}(x), \quad \beta=\frac{2 N}{g_{0}^{2}},
\end{aligned}
$$

where $g_{0}$ is the bare coupling, $\Pi_{\mu \nu}$ is the standard plaquette operator, given by the product of link variables along a $1 \times 1$ plaquette of the lattice

$$
\Pi_{\mu \nu}(x)=U_{\mu}(x) U_{\nu}(x+\mu+\nu) U_{\mu}^{\dagger}(x+\nu) U_{\nu}^{\dagger}(x),
$$

and the topological term

$$
Q_{L} \equiv \sum_{x} q_{L}(x)
$$

is constructed using the lattice operator 21]

$$
q_{L}(x)=-\frac{1}{2^{4} \times 32 \pi^{2}} \sum_{\mu \nu \rho \sigma= \pm 1}^{ \pm 4} \epsilon_{\mu \nu \rho \sigma} \operatorname{Tr}\left[\Pi_{\mu \nu} \Pi_{\rho \sigma}\right] .
$$

Notice that this is not the only possible choice for $q_{L}$; the only requirement is that it must have the correct continuum limit (2) when $a \rightarrow 0$ ( $a$ is the lattice spacing). 
Our choice of the operator $q_{L}(x)$ is also motivated by its relative simplicity in view of the MC simulations. In the continuum limit 22] $q_{L}(x)$, being a local operator, behaves as

$$
q_{L}(x) \longrightarrow a^{4} Z_{q} q(x)+O\left(a^{6}\right),
$$

where $Z_{q}$ is a finite function of the bare coupling, going to one in the limit $\beta \rightarrow \infty$. Thus, we have the correspondence

$$
\theta_{i}=Z_{q} \theta_{L}
$$

apart from $O\left(a^{2}\right)$ corrections. The renormalization $Z_{q}$ may be evaluated by $\mathrm{MC}$ simulation at $\theta=0$, computing

$$
Z_{q}=\frac{\left\langle Q Q_{L}\right\rangle_{\theta=0}}{\left\langle Q^{2}\right\rangle_{\theta=0}}
$$

where $Q$ is a topological estimator such as those obtained by the overlap method [23, 24] or the cooling method [25], which are not affected by lattice renormalizations, and, more importantly, by nonphysical background contributions arising when the correlations of topological charge densities are measured at coincident points. Note that Eq. (21) assumes that such contributions are also absent when we consider expectation values of mixed products of $Q$ and $Q_{L}$; this hypothesis will be checked by the scaling consistency of the results. In particular, we expect that the ratios

$$
\begin{aligned}
& \frac{\langle Q\rangle_{\theta_{i}}}{\left\langle Q^{2}\right\rangle_{\theta=0}}=\theta_{i}-2 b_{2} \theta_{i}^{3}+3 b_{4} \theta_{i}^{5}+\ldots, \\
& \frac{\left\langle Q^{2}\right\rangle_{\theta_{i}}^{c}}{\left\langle Q^{2}\right\rangle_{\theta=0}}=1-6 b_{2} \theta_{i}^{2}+15 b_{4} \theta_{i}^{4}+\ldots,
\end{aligned}
$$

have a well defined continuum limit as functions of $\theta_{i}$.

We mention that similar approaches based on $\mathrm{MC}$ simulations with imaginary $\theta$ terms have been also pursued to study the $\theta$ dependence of $2 \mathrm{D} \mathrm{CP}^{N-1}$ models, see Refs. 26, 27, 28, 29].

In the following we present results of MC simulations of the $4 \mathrm{D} \mathrm{SU}(3)$ lattice gauge theory, at $\beta=5.9,6,6.2$, for lattice sizes $L=16,16,20$, respectively; the simulations are carried out both at $\theta_{L}=0$ and $\theta_{L} \neq 0$, within the region $\left|\theta_{i}\right| \lesssim \pi$. The updating of the link variables is performed using the overrelaxation algorithm described in Ref. 30]. Since our numerical study requires high-statistics MC simulations, we choose the cooling method as estimator of the topological charge $Q$, and in particular the implementation outlined in Ref. 15]. The topological charge has been measured on cooled configurations (by locally minimizing the lattice action), using the twisted double plaquette operator (18). As is well known, the sum over the whole lattice of the twisted double plaquette, measured on cooled configurations, takes on values $Q_{t} \simeq k \alpha$, where $k$ is an integer and $\alpha \lesssim 1$. We determine the typical value of 


\begin{tabular}{cclccc}
\hline \hline$\beta$ & $L$ & $\chi \equiv\left\langle Q^{2}\right\rangle_{\theta=0} / V$ & $b_{2}$ & $b_{4}$ & $Z_{q}$ \\
\hline \hline 5.9 & 16 & $0.0001532(4)$ & $-0.026(6)$ & $-0.016(13)$ & $0.1122(6)$ \\
6.0 & 16 & $0.0000743(2)$ & $-0.027(4)$ & $0.004(3)$ & $0.1353(9)$ \\
6.2 & 20 & $0.0000201(1)$ & $-0.028(4)$ & $0.000(2)$ & $0.174(2)$ \\
\hline \hline
\end{tabular}

Table 1: $\theta=0 \mathrm{MC}$ results for the $4 \mathrm{D} \mathrm{SU}(3)$ lattice gauge theory.
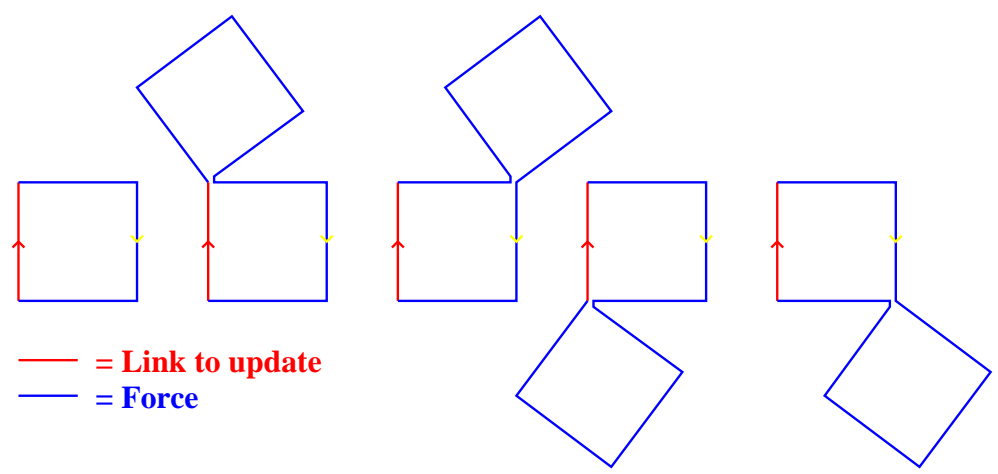

Figure 1: The force corresponding to a given link. All but the first contribution arise from the $\theta$ term.

$\alpha$ by minimizing the average deviation of the twisted double plaquette from integer multiples of $\alpha$, and then assign to $Q$ the integer closest to $Q_{t} / \alpha$. The factor $\alpha$ turns out to depend on $\beta$, and approaches one with increasing $\beta$; for example $\alpha \approx 0.93$ for $\beta=6$ and $\alpha \approx 0.95$ for $\beta=6.2$, at the $20^{\text {th }}$ cooling step. This method eliminates the need for expensive, protracted cooling; usually fewer than 20 steps suffice (when using $N(N-1) / 2$ subgroups) in order to observe a substantial convergence of the results, which appears to improve with increasing $\beta$. The data that we will report are taken after 20 cooling steps. This cooling method to estimate $Q$ is significantly less expensive than the overlap method, although less rigorous [4].

To begin with, we report results from high-statistics $\mathrm{MC}$ simulations at $\theta=0$. They required long MC runs, essentially because of the severe (apparently exponential) critical slowing down of the topological modes [15], whose autocorrelation time (in units of sweeps) ranges from $\tau_{Q} \approx 77$ at $\beta=6$ to $\tau_{Q} \approx 540$ at $\beta=6.2$. In particular, over 40 million sweeps per value of $\beta$ were produced on average for runs at $\theta=0$. The results for the topological susceptibility $\chi$, the coefficients $b_{2}$ and $b_{4}$, and the renormalization constant $Z_{q}$, are reported in Table 11. The estimates of $\chi$ and $b_{2}$ are consistent with earlier MC simulations, see, e.g., [15]. The estimate of high-order coefficients, such as $b_{4}$, turns out to be very hard in $\theta=0 \mathrm{MC}$ simulations, requiring huge statistics, due to the large cancellations in their expressions in terms of correlators at $\theta=0$, see $\mathrm{Eq}$ (9), giving rise to relatively large statistical errors. The results for $b_{4}$ are consistent with zero, suggesting the bound $\left|b_{4}\right| \lesssim 0.005$, which will be improved below. The estimates of $Z_{q}$ may be compared with the one-loop 


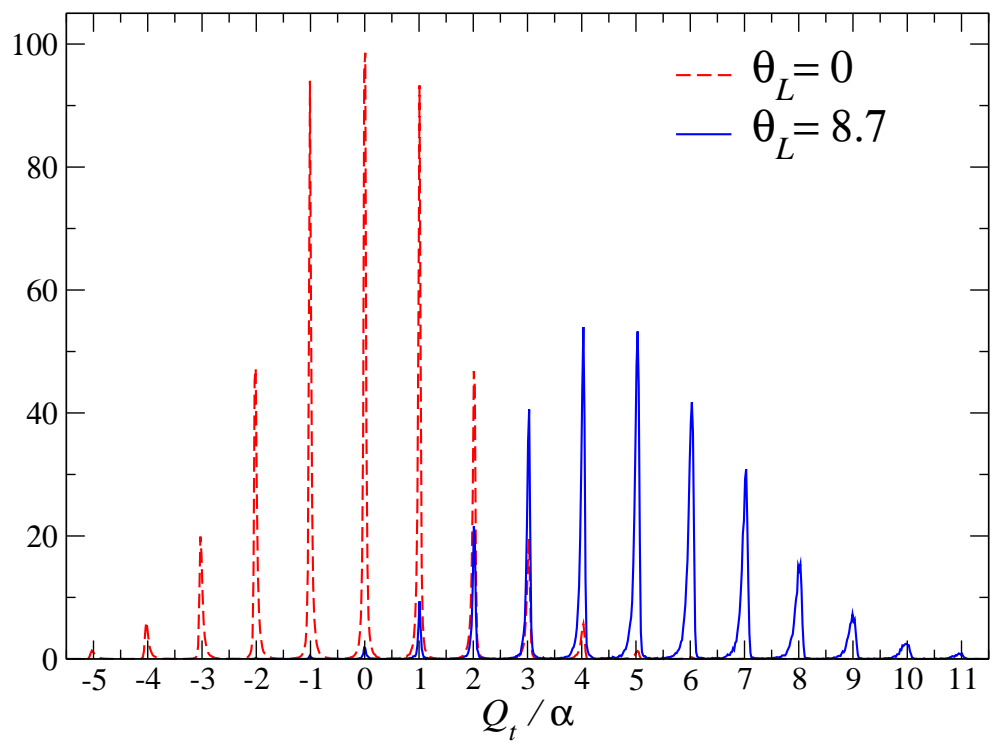

Figure 2: Distribution of the ratio $Q_{t} / \alpha$, for $\beta=6.2$ configurations at $\theta_{L}=0$ and $\theta_{L}=8.7$ (corresponding to $\theta_{i} \approx 1.5$ ), after 20 cooling steps.

calculation 22 $Z_{q}=1-5.448 \beta^{-1}+O\left(\beta^{-2}\right)$, which underestimates it, and the estimates by the heating method at $\beta=6: Z_{q}=0.18(1)$ [31] and $Z_{q}=0.16(1)$ [17], which are slightly larger than the values that we obtain in our MC simulations using Eq. (21).

The MC simulations at $\theta_{L} \neq 0$ are quite slower, due to the more complex structure of the action terms involving a single link. The "force" corresponding to a given link now consists of additional contributions, beyond the standard sum of staples, as shown in Fig. 1. Thus, the link updating procedure is slower by approximately a factor of three with respect to the $\theta_{L}=0$ case. In runs with $\theta_{L} \neq 0$, an average of 3 million sweeps were produced for each value of $\beta$ and $\theta_{L}$.

The cooling algorithm is implemented in the same way as for the $\theta_{L}=0$ simulations. The presence of a $\theta_{L}$ term with $\theta_{L}>0\left(\theta_{L}<0\right)$ leads to a preponderance of configurations with $Q>0(Q<0)$. Fig. 2 shows the distributions of the ratio $Q_{t} / \alpha$ of $\beta=6.2$ cooled configurations at $\theta_{L}=0$ and $\theta_{L}=8.7$ (corresponding to $\theta_{i}=Z_{q} \theta_{L} \approx 1.5$ ), after 20 cooling steps; we recall that the topological estimator $Q$ is obtained by taking the integer closest to $Q_{t} / \alpha, \alpha(\beta=6.2)=0.95$. We note that these distributions cluster around integer values, also for rather large values of $Q_{t} / \alpha$, both for $\theta_{L}=0$ and $\theta_{L}=8.7$, although the optimal value $\alpha=0.95$ is kept fixed. The autocorrelation time of the topological charge in the MC simulations at $\theta_{L} \neq 0$ does not significantly change in units of sweeps with respect to that at $\theta_{L}=0$ and the same $\beta$.

Figs. 3 and 4 show MC results for the ratios $\langle Q\rangle_{\theta_{L}} /\left\langle Q^{2}\right\rangle_{\theta=0}$ and $\langle Q\rangle_{\theta_{L}} /\left\langle Q^{2}\right\rangle_{\theta_{L}}^{c}$, respectively, versus $\theta_{i}=Z_{q} \theta_{L}$. The MC data at different $\beta$ values appear to follow the 


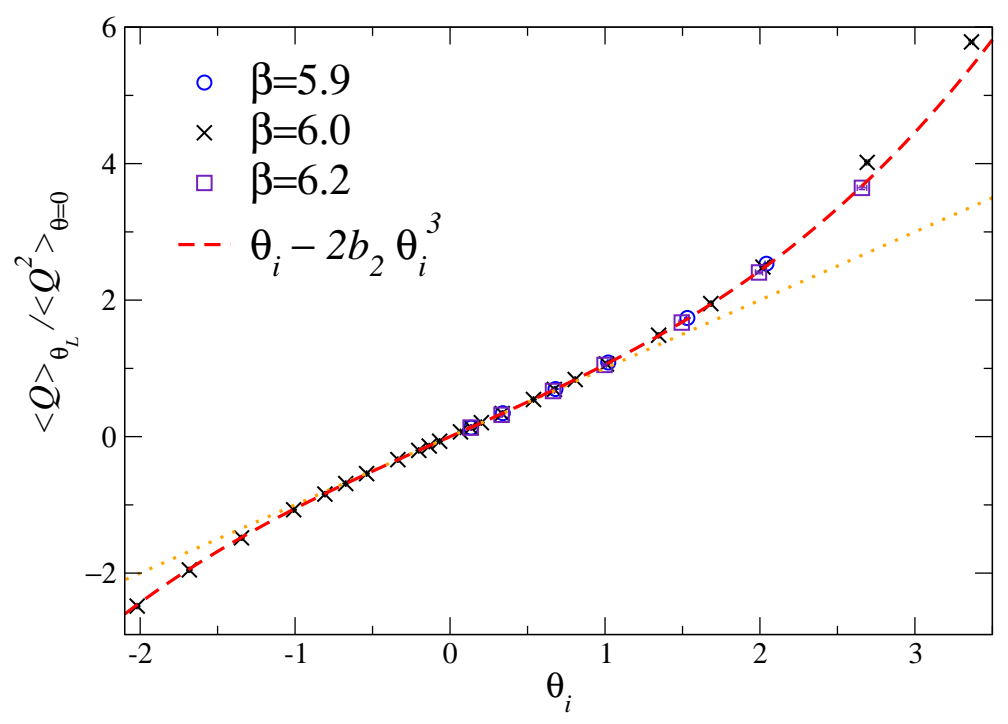

Figure 3: The ratio $\langle Q\rangle_{\theta_{L}} /\left\langle Q^{2}\right\rangle_{\theta=0}$ vs $\theta_{i}=Z_{q} \theta_{L}$. Here scaling corrections are only visible for $\theta_{i}>2$. The dashed line shows the curve $\theta_{i}-2 b_{2} \theta_{i}^{3}$ with $b_{2}=-0.026$, while the dotted line shows the simple linear behavior.

same curve, providing evidence of scaling. Scaling corrections, which are expected to be $O\left(a^{2}\right)$, appear quite small, and tend to increase with increasing $\theta_{i}$. This good scaling behavior thus corroborates the existence of a nontrivial continuum limit for any value of $\theta_{i}$.

In Table 2 we present the results of fits of the data for the ratio $\langle Q\rangle_{\theta_{L}} /\left\langle Q^{2}\right\rangle_{\theta=0}$, to the polynomial Ansatz

$$
\frac{\langle Q\rangle_{\theta_{L}}}{\left\langle Q^{2}\right\rangle_{\theta=0}}=Z_{q} \theta_{L}-2 b_{2}\left(Z_{q} \theta_{L}\right)^{3}+3 b_{4}\left(Z_{q} \theta_{L}\right)^{5}+\ldots
$$

The results turn out to be quite stable with respect to the maximum value of $\left|\theta_{i}\right|$ allowed in the fits. They are in good agreement with the $\theta=0$ results presented in Table 1, improving them significantly. In particular, they provide a much smaller bound on the value of $b_{4}$. The expected $O\left(a^{2}\right)$ scaling corrections in the estimates of $b_{2 i}$ are taken into account by extrapolating the results at finite $\beta$ fitting them to

$$
b_{2 i}+c \sigma(\beta)
$$

where $\sigma$ is the string tension in unit of the lattice spacing (we use the data of Ref. [15). This provides an accurate estimate of $b_{2}$, that is

$$
b_{2}=-0.026(3) \text {, }
$$

whose error includes statistical and systematic errors related to the extrapolation to the continuum limit and to the small differences of the fit results reported in Table 2. This estimate of $b_{2}$ is clearly more precise than the one we may obtain 


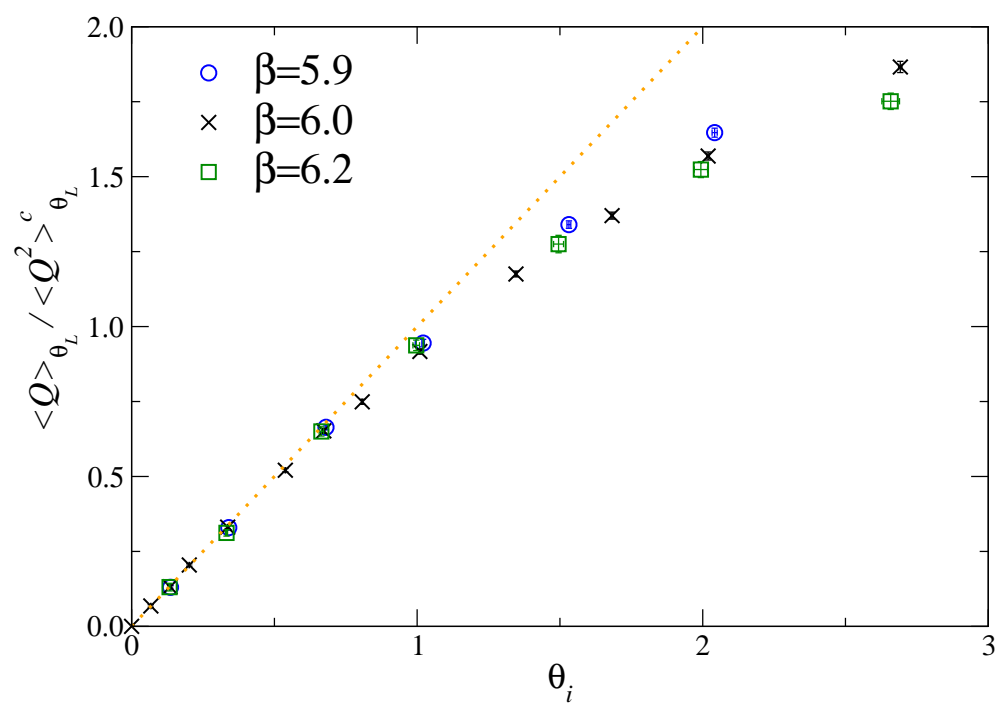

Figure 4: The ratio $\langle Q\rangle_{\theta_{L}} /\left\langle Q^{2}\right\rangle_{\theta_{L}}^{c}$ vs $\theta_{i}=Z_{q} \theta_{L}$.

by a continuum-limit extrapolation of the data at $\theta=0$ reported in Table 1 using Eq. (25), which gives $b_{2}=-0.029(7)$. Moreover, the results of Table 2 lead to a very small bound for $b_{4}$ :

$$
\left|b_{4}\right|<0.001
$$

which is much smaller than the one obtained from the $\theta=0$ simulations. These results confirm that the coefficients $b_{2 i}$ of the expansion (11) are rapidly decreasing with increasing $i$, as expected by the large- $N$ prediction $b_{2 i}=O\left(N^{-2 i}\right)$ [ [

The results (26) and (27) significantly improve earlier results for the expansion of the ground-state energy around $\theta=0$, as obtained by $\theta=0 \mathrm{MC}$ simulations using different methods to estimate $Q: b_{2}=-0.023(7)$ from Ref. [15], $b_{2}=-0.024(6)$ from Ref. [17], and $b_{2}=-0.025(9)$ from Ref. [18], while no estimates for $b_{4}$ had been reported.

In conclusion, we have investigated the scaling behavior of the $4 \mathrm{D} \mathrm{SU}(3)$ gauge theory in the presence of an imaginary $\theta$ term, by MC simulations computing the topological properties at imaginary $\theta$, i.e. $\theta=-i \theta_{i}$ with real $\theta_{i}$. The numerical results for the topological charge provide a good evidence of scaling in the region $\left|\theta_{i}\right| \lesssim \pi$ which we consider. The imaginary $\theta$ dependence of the ground-state energy turns out to be well described by the first few nontrivial terms of the expansion around $\theta=0$. Fits to polynomials provide a quite accurate estimate of $b_{2}$ and a very small bound on $b_{4}$, see Eqs. (26) and (27), which support the expected large- $N$ scenario predicting $b_{2 i}=O\left(N^{-2 i}\right)$. This study may be straightforwardly extended to other observables, to determine their $\theta$ dependence, see Ref. [32]. Finally, we mention that, besides allowing more precise determinations of the $\theta=0$ expansion coefficients of the ground-state energy and other observables, the use of imaginary $\theta$ values might 


\begin{tabular}{ccccll}
\hline \hline$\beta$ & order of the polynomial & $\left|\theta_{i}\right|_{\max }$ & \multicolumn{1}{c}{$Z_{q}$} & \multicolumn{1}{c}{$b_{2}$} & $b_{4}$ \\
\hline \hline 5.9 & 1.0 & $0.1121(5)$ & $-0.030(3)$ & \\
& $O\left(\theta_{L}^{3}\right)$ & 1.5 & $0.1122(4)$ & $-0.0285(14)$ & \\
& 2.0 & $0.1122(3)$ & $-0.0287(8)$ & \\
& & 1.0 & $0.1120(5)$ & $-0.025(6)$ & $0.004(4)$ \\
& & 1.5 & $0.1122(4)$ & $-0.029(4)$ & $0.000(1)$ \\
& & 2.0 & $0.1122(4)$ & $-0.029(2)$ & $0.0000(3)$ \\
\hline 6 & $O\left(\theta_{L}^{3}\right)$ & 1.0 & $0.1346(4)$ & $-0.028(2)$ & \\
& & 1.3 & $0.1346(3)$ & $-0.0285(14)$ & \\
& & 1.7 & $0.1346(3)$ & $-0.0282(9)$ & \\
& & 2.0 & $0.1346(3)$ & $-0.0282(6)$ & \\
& & 1.0 & $0.1345(4)$ & $-0.027(4)$ & $0.002(3)$ \\
& & 1.3 & $0.1346(4)$ & $-0.028(3)$ & $0.000(1)$ \\
& & 1.7 & $0.1346(4)$ & $-0.028(2)$ & $0.0000(5)$ \\
& & 2.0 & $0.1346(4)$ & $-0.028(2)$ & $0.0000(2)$ \\
\hline 6.2 & $O\left(\theta_{L}^{5}\right)$ & 1.0 & $0.1717(13)$ & $-0.027(4)$ & \\
& & 1.5 & $0.1716(13)$ & $-0.027(3)$ & \\
& & 2.0 & $0.1717(13)$ & $-0.027(2)$ & \\
& & 2.7 & $0.1717(12)$ & $-0.0265(13)$ & \\
& & 1.0 & $0.1724(15)$ & $-0.027(4)$ & $-0.002(6)$ \\
& & 1.5 & $0.1722(13)$ & $-0.027(4)$ & $-0.0003(14)$ \\
& & 2.0 & $0.1722(13)$ & $-0.027(3)$ & $-0.0002(6)$ \\
& & 2.7 & $0.1721(12)$ & $-0.026(3)$ & $-0.0000(2)$ \\
\hline \hline
\end{tabular}

Table 2: Results of some fits of the data of $\langle Q\rangle_{\theta_{L}} /\left\langle Q^{2}\right\rangle_{\theta=0}$ to $O\left(\theta_{L}^{k}\right)$ odd polynomials. The fits use also the $\theta=0$ results of Table 1 and consider only data up to a maximum value $\left|\theta_{i}\right|_{\max }$ of $\left|\theta_{i}\right|$, leading to an acceptable $\chi^{2}, \chi^{2} /$ dof $\lesssim 2$.

turn out useful in the effort to overcome the problem of the dramatic critical slowing down of the topological modes 4 , 15, 33, 34, 35], by performing parallel tempering simulations [36] with a set of imaginary $\theta$ values including $\theta=0$, which provides an exact $\mathrm{MC}$ algorithm for the model at $\theta=0$. This approach is largely used in the MC simulations of spin-glass models which are affected by analogous critical slowing down problems.

\section{References}

[1] G. 't Hooft, A planar diagram theory for strong interactions, Nucl. Phys. B 72 (1974) 461.

[2] E. Witten, Instantons, the quark model, and the $1 / N$ expansion, Nucl. Phys. B 149 (1979) 285.

[3] E. Witten, Large-N chiral dynamics, Ann. Phys. (NY) 128 (1980) 363. 
[4] E. Vicari and H. Panagopoulos, $\theta$ dependence of $S U(N)$ gauge theories in the presence of a topological term, Phys. Rep. 470 (2009) 93 [arXiv:0803.1593 hep-th].

[5] C.A. Baker, D.D. Doyle, P. Geltenbort, K. Green, M.G.D. van der Grinten, P.G. Harris, P. Iaydjiev, S.N. Ivanov, D.J.R. May, J.M. Pendlebury, J.D. Richardson, D. Shiers, K.F. Smith, An improved experimental limit on the electric dipole moment of the neutron, Phys. Rev. Lett. 97 (2006) 131801 [arXiv:hep-ex/0602020].

[6] P.G. Harris, C.A. Baker, K. Green, P. Iaydjiev, S. Ivanov, D.J.R. May, J.M. Pendlebury, D. Shiers, K.F. Smith, M. van der Grinten, and P. Geltenbort, New experimental limit on the electric dipole moment of the neutron, Phys. Rev. Lett. 82 (1999) 904.

[7] G. 't Hooft, Symmetry breaking through Bell-Jackiw anomalies, Phys. Rev. Lett. 37 (1976) 8.

[8] E. Witten, Current algebra theorems for the U(1) "Goldstone boson", Nucl. Phys. B 156 (1979) 269.

[9] G. Veneziano, U(1) without instantons, Nucl. Phys. B 159 (1979) 213.

[10] O. Aharony, S.S. Gubser, J.M. Maldacena, H. Ouguri, and Y. Oz, Large N field theories, string theory and gravity, Phys. Rep. 323 (2000) 183 [arXiv:hep-th/9904017].

[11] E. Witten, Theta dependence in the large- $N$ limit of four-dimensional gauge theories, Phys. Rev. Lett. 81 (1998) 2862 [arXiv:hep-th/9807109].

[12] P. Di Vecchia, Large $N$ gauge theories and AdS/CFT correspondence, arXiv:hep-th/9908148.

[13] U. Gürsoy and E. Kiritsis, Exploring improved holographic theories for QCD: Part I, JHEP 02 (2008) 032 [arXiv:0707.1324 hep-th].

[14] U. Gürsoy, E. Kiritsis, and F. Nitti, Exploring improved holographic theories for QCD: Part II, JHEP 02 (2008) 019 [arXiv:0707.1349 hep-th].

[15] L. Del Debbio, H. Panagopoulos, and E. Vicari, $\theta$ dependence of $S U(N)$ gauge theories, JHEP 08 (2002) 044 [arXiv:hep-th/0204125].

[16] M. Lüscher, Topological effects in $Q C D$ and the problem of short-distance singularities, Phys. Lett. B 593 (2004) 296 [arXiv:hep-th/0404034].

[17] M. D'Elia, Field approach to the study of the $\theta$ dependence in Yang-Mills theories on the lattice, Nucl. Phys. B 661 (2003) 139 [arXiv:hep-lat/0302007]; private communication.

[18] L. Giusti, S. Petrarca, and B. Taglienti, Theta dependence of the vacuum energy in the SU(3) gauge theory from the lattice, Phys. Rev. D 76 (2007) 094510 [arXiv:0705.2352 hep-th]. 
[19] D. Espriu and R. Tarrach, Renormalization of the axial anomaly operators, Z. Phys. C 16 (1982) 77.

[20] I. Montvay and G. Münster, Quantum Fields on a Lattice, Cambridge University Press (1994).

[21] P. Di Vecchia, K. Fabricius, G.C. Rossi, and G. Veneziano, Preliminary evidence for $U_{A}(1)$ breaking in QCD from lattice calculations, Nucl. Phys. B 192 (1981) 392.

[22] M. Campostrini, A. Di Giacomo, and H. Panagopoulos, The topological susceptibility on the lattice, Phys. Lett. B 212 (1988) 206.

[23] H. Neuberger, Exact chiral symmetry on the lattice, Ann. Rev. Nucl. Part. Sci. 51 (2001) 23 [arXiv:hep-lat/0101006].

[24] P. Hasenfratz, V. Laliena, and F. Niedermayer, The index theorem in QCD with a finite cut-off, Phys. Lett. B 427 (1998) 125 [arXiv:hep-lat/9801021].

[25] M. Teper, Topology in QCD, Nucl. Phys. B (Proc. Suppl.) 83 (2000) 146 [arXiv:hep-lat/9909124].

[26] V. Azcoiti, G. Di Carlo, A. Galante, and V. Laliena, New proposal for numerical simulations of $\theta$-vacuum like systems, Phys. Rev. Lett. 89 (2002) 141601 [arXiv:hep-lat/0203017].

[27] V. Azcoiti, G. Di Carlo, A. Galante, and V. Laliena, Theta dependence of $C P^{9}$ model, Phys. Rev. D 69 (2004) 056006 [arXiv:hep-lat/0305022].

[28] V. Azcoiti, A. Galante, and V. Laliena, Critical behavior of $C P^{1}$ at $\theta=\pi$, Haldane's conjecture and the relevant universality class, Phys. Rev. Lett. 98 (2007) 257203 [arXiv:0710.1507 hep-lat].

[29] B. Allés and A. Papa, Mass gap in the 2D O(3) non-linear sigma model with a $\theta=\pi$ term, Phys. Rev. D 77 (2008) 056008 [arXiv:0711.1496 cond-mat].

[30] L. Del Debbio, H. Panagopoulos, P. Rossi, and E. Vicari, Spectrum of confining strings in $S U(N)$ gauge theories, JHEP 01 (2001) 009 [arXiv:hep-th/0111090].

[31] B. Allés, M. Campostrini, L. Del Debbio, A. Di Giacomo, H. Panagopoulos, and E. Vicari, The proton matrix element of the topological charge in quenched $Q C D$, Phys. Lett. B 336 (1994) 248 [arXiv:hep-lat/9402019].

[32] L. Del Debbio, G. Manca, H. Panagopoulos, A. Skouroupathis, and E. Vicari, $\theta$ dependence of the spectrum of $S U(N)$ gauge theories, JHEP 06 (2006) 005 [arXiv:hep-th/0603041].

[33] L. Del Debbio, G. Manca, and E. Vicari, Critical slowing down of topological modes, Phys. Lett. B 594 (2004) 315 [arXiv:hep-lat/0403001]. 
[34] M. Campostrini, P. Rossi, and E. Vicari, Monte Carlo simulations of $C P^{N-1}$ models, Phys. Rev. D 46 (1992) 2647.

[35] ALPHA Collaboration (S. Schaefer et al.) Critical slowing down and error analysis in lattice QCD simulations, Nucl. Phys. B 845 (2011) 93 [arXiv:1009.5228 hep-lat].

[36] D. J. Earl and M. W. Deem, Parallel tempering: Theory, applications, and new perspectives, Phys. Chem. Chem. Phys. 7 (2005) 3910 [arXiv:physics/0508111]. 